\title{
Marcadores Bioquímicos do Primeiro Trimestre e Recém-Nascidos Leves para Idade Gestacional
}

\author{
First-Trimester Biochemical Markers and Small-for-Gestational-Age Infants
}

\author{
Cláudia ANDRADE ${ }^{1}$, Joana SANTOS ${ }^{1}$, Ana RITA PINTO ${ }^{1}$, Pedro MANSO $^{1}$, Susana PEREIRA ${ }^{1}$ \\ Acta Med Port 2014 Mar-Apr;27(2):191-195
}

\section{RESUMO}

Introdução: Estudos anteriores mostram uma relação dos marcadores bioquímicos do $1^{\circ}$ trimestre, proteína plasmática A associada à gravidez e subunidade $\beta$ da gonadotrofina coriónica, com o nascimento de recém-nascidos com peso abaixo do percentil 10 . 0 nosso objectivo foi descrever a relação entre estes marcadores bioquímicos com os recém-nascidos leves para a idade gestacional, na nossa população.

Material e Métodos: Estudo retrospectivo analítico de 2305 grávidas que realizaram o rastreio combinado do primeiro trimestre entre Março 2009 e Setembro de 2011. Comparação entre o grupo dos recém-nascidos abaixo do percentil 10 e o grupo controlo (recémnascidos de termo com peso acima do percentil 10) e os recém-nascidos abaixo do percentil 3 e o grupo controlo. Foi realizado uma análise de regressão múltipla e logística com a utilização dos valores de proteína plasmática $A$ associada à gravidez e subunidade $\beta$ da gonadotrofina coriónica (em múltiplos da mediana) e as características demográficas maternas como etnia, peso e status tabágico. Resultados: O estudo revelou uma contribuição independente da proteína plasmática A associada à gravidez, do peso materno e dos hábitos tabágicos para os recém-nascidos abaixo do percentil 10 . Na regressão logística para o marcador proteína plasmática A associada à gravidez, o risco relativo abaixo do percentil 10 foi de 2,41 e abaixo do percentil 3 de 3,41 ( $p<0,01$ ). No caso da subunidade $\beta$ da gonadotrofina coriónica, o odds ratio determinado para percentil inferior a 10 foi de $1,70(p=0,03)$ e para o percentil inferior a 3 foi de $3,22(p<0,01)$.

Conclusões: Baixos níveis da proteína plasmática $A$ associada à gravidez e de subunidade $\beta$ da gonadotrofina coriónica (valores inferiores ao percentil 5 da população estudada) estiveram relacionados com aumento do risco do nascimento de recém-nascidos leves para a idade gestacional na população de grávidas abrangidas pelo estudo.

Palavras-chave: Diagnóstico Prenatal; Marcadores Biológicos; Portugal; Primeiro Trimestre da Gravidez; Recém-nascido Leve para a Idade Gestacional.

\section{ABSTRACT}

Introduction: Several studies suggested an association between first trimester biochemical markers (PAPP-A and $\beta-H C G)$ and infants below $10^{\text {th }}$ percentile. Our goal was to describe this relationship of biochemical markers with small-for-gestational-age fetuses in our population.

Material and Methods: Retrospective analytic study of 2035 pregnant women that underwent first-trimester screening in the period between March 2009 and September 2011. Small-for-gestational-age infants below $10^{\text {th }}$ percentile were compared with control group (term newborn with birth weight above $10^{\text {th }}$ percentile). Infants below $3^{\text {rd }}$ percentile and control group were also compared. Multiple and logistic regression analysis were done with PAPP-A, $\beta$-HCG (multiples of the expected normal median) and demographic maternal characteristics (ethnicity, weight and smoker status).

Results: This study demonstrated an independent contribution of PAPP-A, maternal weight and smoker status in predicting smallfor-gestational-age infants. For PAPP-A, the odds ratio for small-for-gestational age below $10^{\text {th }}$ and $3^{\text {rd }}$ percentile was 2.41 and 3.41 , respectively $(p<0.01)$. For $\beta-\mathrm{HCG}$, odds ratio below $10^{\text {th }}$ percentile was $1.70(p=0.03)$ and for birth weight below the 3rd percentile, the odds ratio was $3.22(p<0.01)$.

Conclusions: Low levels of PAPP-A and $\beta-$ HCG (values below 5th percentile of the study population) were associated with an increased risk of small-for-gestational-age infants in the pregnant population included in this study.

Keywords: Biological Markers; Infant, Small for Gestational Age; Pregnancy Trimester, First; Prenatal Diagnosis; Portugal.

\section{INTRODUÇÃO}

Nos últimos anos tem-se assistido a um aumento de evidência das potencialidades dos marcadores bioquímicos do primeiro trimestre como marcadores de resultados gestacionais adversos, entre eles, recém-nascidos leves para a idade gestacional (LIG), partos prematuros, mortes fetais in utero, abortamentos e pré-eclâmpsia. ${ }^{1-10} \mathrm{~A}$ capacidade de detecção precoce de gestações em risco aumentado de morbilidade e mortalidade fetal, tem uma importante implicação clínica de modo a optimizar a vigilância pré-natal. ${ }^{1,11}$

A proteína plasmática $A$ associada à gravidez (PAPP-A) é uma proteína derivada do trofoblasto que actua como protease na ligação de factores de crescimento insulin-like e que, em termos teóricos, é responsável pela estimulação de factores crescimento placentares. ${ }^{12,13}$ Estudos anteriores mostram que níveis baixos de PAPP-A estão associados, como factor independente, a um risco aumentado de recém-nascidos leves para a idade gestacional (LIG) $)^{1-3,5,11,14}$ ou seja recém-nascidos com peso inferior ao percentil 10. A subunidade $\beta$ da gonadotrofina coriónica humana ( $\beta-\mathrm{HCG}$ ) como marcador bioquímico do primeiro trimestre, também se encontra associada a estes recém-nascidos LIG quando se apresenta com valores baixos. ${ }^{1,3,5,11,14}$

\footnotetext{
1. Departamento de Obstetrícia e Ginecologia. Centro Hospitalar Tondela-Viseu. Viseu. Portugal.

Recebido: 19 de Abril de 2013 - Aceite: 06 de Novembro de 2013 | Copyright $\odot$ Ordem dos Médicos 2014
} 
O objectivo deste trabalho foi demonstrar a relação dos marcadores bioquímicos do primeiro trimestre (PAPP-A e $\beta-\mathrm{HCG}$ ) rastreados prospectivamente na nossa população, com a respectiva capacidade de prever recém-nascidos leves para a idade gestacional.

\section{MATERIAL E MÉTODOS}

Estudo de coorte retrospectivo do processo clínico de todas as grávidas que realizaram o rastreio combinado do primeiro trimestre, na nossa Unidade de Medicina Fetal, no Centro Hospitalar Tondela-Viseu, no período compreendido entre Março de 2009 e Setembro de 2011.

$\mathrm{O}$ rastreio combinado do primeiro trimestre realizado a todas as grávidas incluiu a colheita de sangue periférico entre as $9^{+0}$ e $13^{+6}$ semanas de gestação para a determinação dos valores de PAPP-A e $\beta$-HCG seguida de ecografia fetal entre as $11^{+0}$ e $13^{+6}$ semanas para avaliação do comprimento crâneo-caudal ( $C R L)$, da translucência da nuca (TN) e marcadores de risco adicional, quando indicado. ${ }^{15}$

As medidas analíticas séricas de PAPP-A e $\beta$-HCG foram convertidas em valores múltiplos da mediana (MoM) ajustados à idade gestacional, etnia, peso materno e tabagismo. ${ }^{16}$ Durante a análise estatística, as variáveis PAPP-A e $\beta$-HCG corresponderam não só a variáveis contínuas como dicotómicas, tendo para isso sido utilizados valores de cut-off correspondentes ao percentil 5 da população estudo (0,439 MoM para PAPP-A e 0,313 MoM para $\beta-H C G)$.

Os dados demográficos (idade materna e paridade) e

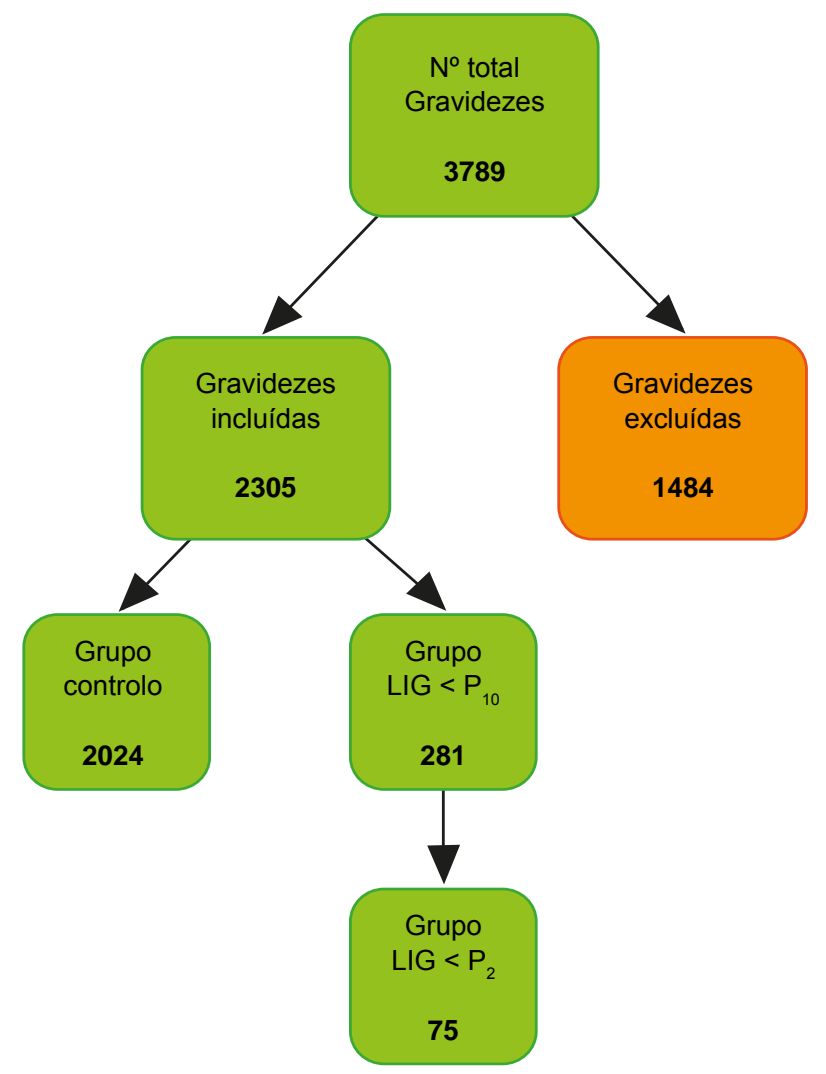

Figura 1 - Fluxograma descritivo do número de casos utilizados.

(LIG - leve para a idade gestacional; $P_{10}-$ percentil $10 ; P_{3}$ - percentil 3) a história médica (hábitos tabágicos e peso materno), coIhidos na primeira visita à nossa unidade, assim como o registo do desfecho neonatal (idade gestacional do parto, peso do nascimento), foram registados numa base de dados computorizada de acesso restrito (PIA-Fetal Database, ViewPoint, Webling, Alemanha). A idade (em anos), paridade (se é nulípara ou não) e o peso materno (em quilogramas) foram registados na altura da colheita de sangue. Os hábitos tabágicos foram classificados como 'fumadora' e 'não fumadora' independentemente do número de cigarros/ dia consumidos, na altura da colheita de sangue. Os dados dos partos e dos recém-nascidos nascidos fora do nosso hospital foram obtidos através de contacto telefónico com as parturientes.

Foram excluídos casos de gravidez múltipla, fetos com alterações cromossómicas ou estruturais major, fetos com idade gestacional inferior ou igual a 25 semanas, prematuros com peso acima do percentil 10 e casos com dados incompletos.

As 2305 gravidezes registadas na base de dados foram divididas em dois grandes grupos: um grupo controlo constituído pelos recém-nascidos de termo (depois das 37 semanas) com peso igual ou superior ao percentil 10 . O grupo de estudo, designado por grupo dos recém-nascidos leves para idade gestacional (LIG), correspondeu a todos os recém-nascidos com peso à nascença inferior ao percentil $10 .{ }^{17} \mathrm{O}$ grupo dos LIG ainda foi dividido num outro subgrupo correspondente ao peso à nascença inferior ao percentil 3 para a idade gestacional (Fig. 1).

Os dados foram analisados através de uma regressão múltipla para determinar a contribuição independente dos marcadores bioquímicos (em MoM) na determinação dos LIG assim como outras variáveis como o peso materno, a etnia materna e o tabagismo. Além desta análise, foi também realizada uma regressão logística para calcular o risco relativo do PAPP-A e $\beta-H C G$ nos LIG para o percentil 10 e para o percentil 3. O valor de $p$ inferior a 0,05 foi considerado estatisticamente significativo. Análise estatística foi efectuada com o sistema informático SPSS para Windows (versão 18.0, Chicago, IL, USA).

\section{RESULTADOS}

Foram incluídas 2305 grávidas no estudo e excluídos 1484 casos com alterações cromossómicas ou estruturais fetais, gravidezes múltiplas, idades gestacionais inferiores às 25 semanas, prematuros com peso acima do percentil 10 e gravidezes com dados incompletos. Na distribuição pelos grupos de estudo, o grupo controlo correspondeu a 2024 recém-nascidos $(87,8 \%)$ e os restantes 281 recém-nascidos $(12,2 \%)$ corresponderam ao grupo de recém-nascidos com peso inferior ao percentil 10, dos quais 75 recém-nascidos com peso inferior ao percentil 3 (3,3\%). As características demográficas maternas, a idade gestacional na altura do parto, o peso do recém-nascido ao nascimento e o valor médio dos marcadores bioquímicos para cada grupo em estudo estão representados na Tabela 1. A média registada do marcador PAPP-A foi significativamente infe- 
Tabela 1 - Características demográficas maternas e gestacionais e marcadores bioquímicos

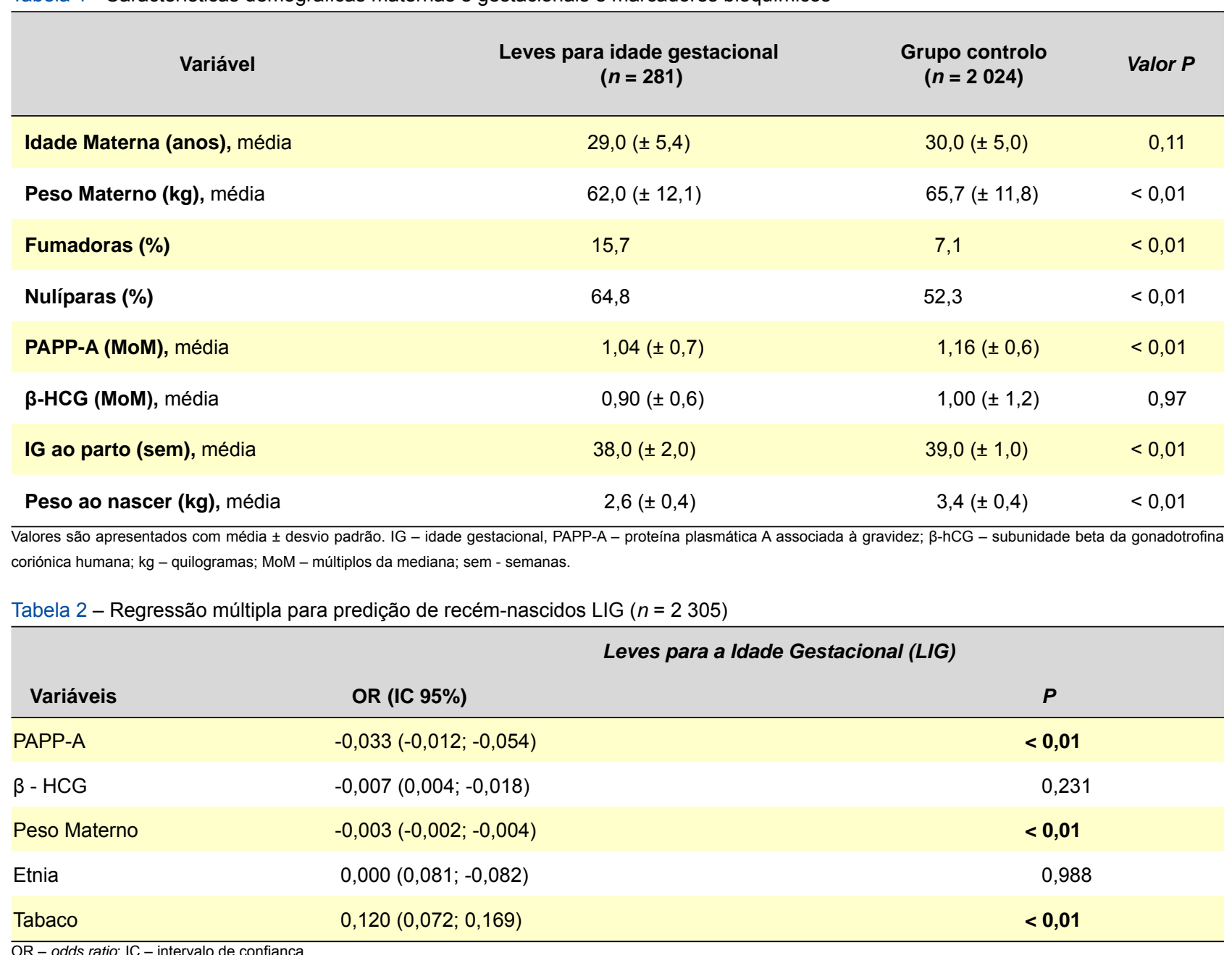

OR - odds ratio; IC - intervalo de confiança

Tabela 3 - Regressão logística para a comparação da média de MoM da PAPP-A e $\beta$-hCG nos recém-nascidos LIG;

\begin{tabular}{|c|c|c|c|c|c|c|}
\hline \multirow[b]{2}{*}{ LIG } & \multicolumn{3}{|c|}{ PAPP-A } & \multicolumn{3}{|c|}{$\beta-$ HCG } \\
\hline & MoM & $P$ & OR & MoM & $P$ & OR \\
\hline$<$ P10 & 1,037 & 0,000 & 2,41 & 0,902 & 0,028 & 1,70 \\
\hline < P3 & 0,916 & 0,000 & 3,41 & 0,870 & 0,001 & 3,22 \\
\hline
\end{tabular}

rior no grupo LIG, quer abaixo do percentil 10 [PAPP-A = $1,04 \pm 0,67 ; p<0,01]$ quer no grupo abaixo do percentil 3 [PAPP-A $=0,92 \pm 0,48 ; p=0,01$ ]. Não houve diferenças significativas na média dos dois grupos quanto à $\beta-H C G$ no grupo LIG $[\beta-H C G=0,90 \pm 0,63 ; p=0,97]$. Com a excepção da idade materna, todas as restantes variáveis clínicas estudadas (Tabela 1) tiveram significado estatístico nos dois grupos - o peso materno, o tabagismo, a nuliparidade, a idade gestacional e o peso ao nascimento.

A análise de regressão múltipla revelou uma contribuição independente da PAPP-A, do peso materno e dos hábitos tabágicos para a previsão dos LIG $\left[\mathrm{R}^{2} 0,025 ; p<\right.$ 0,01 ] (Tabela 2). Já no caso da $\beta-H C G$, este marcador não mostrou uma contribuição significativa $(p>0,05)$.

$\mathrm{Na}$ regressão logística, quando o valor de PAPP-A correspondeu ao percentil 5 da população em estudo, o risco relativo para um LIG abaixo do percentil 10 e percentil 3 foram respectivamente 2,41 (OR 2,41; IC 95\%, 1,6 - 3,7) e 3,41 (OR 3,41; IC 95\%, 1,8 - 6,5) com $p<0,01$. Houve igualmente diferenças estatisticamente significativas encontradas para o percentil 5 da população em estudo de $\beta-H C G$, com um risco relativo de 1,70 (OR 1,70; IC 95\%, $1,1-2,7)$ para o percentil inferior a $10(p=0,03)$ e de 3,22 (OR 3,22; IC 95\%, 1,7 - 6,3) para o percentil inferior a 3 $(p=0,00)$ (Tabela 3$)$. 


\section{DISCUSSÃO}

Este estudo mostrou uma incidência aumentada de LIG em mães fumadoras e uma relação inversamente proporcional da incidência de LIG com o peso materno, colocando as mães com menos peso em maior risco de terem recém-nascidos LIG. Além disso, o estudo demonstrou uma associação entre níveis séricos baixos de PAPP-A do primeiro trimestre com recém-nascidos LIG, que fundamenta uma possível hipótese de insuficiência placentar nestes recém-nascidos com peso inferior ao percentil 10.

Os resultados com a $\beta$-HCG foram menos consensuais nesta relação uma vez que não houve diferença estatisticamente significativa no valor médio desta gonadotrofina entre os dois grupos de estudo na análise descritiva. No entanto, encontrámos associação deste marcador, como factor independente, com recém-nascidos LIG, embora com uma força estatística inferior à PAPP-A. O número baixo de gravidezes incluídas no grupo de estudo pode ser uma possível explicação para estes resultados, que apesar de contraditórios são concordantes com estudos publicados anteriormente. $3,5,6,14$

O aumento da utilização dos marcadores bioquímicos no primeiro trimestre levou ao acréscimo de publicações científicas que demostraram igual relação destes marcadores com recém-nascidos LIG, sobretudo a PAPP-A. ${ }^{1-5,11-14} \mathrm{~A}$ Tabela 4 sumariza os dados publicados em alguns destes estudos relativamente ao valor dos marcadores bioquímicos e odds ratio calculados.

Apesar dos resultados concordantes, os valores correspondentes de odds ratio são baixos, significando que, apesar de aumentar a probabilidade de existência de recém-nascidos LIG, a presença de valores baixos destes marcadores não é determinante absoluta para o nascimento destes recém-nascidos. No entanto a probabilidade é maior quanto mais baixo for o percentil do peso associado.
Além da relação com recém-nascidos LIG, estes marcadores têm vindo a ser estudados como factores preditivos de outras complicações obstétricas. Valores baixos de PAPP-A estão também associados ao parto pré-termo, ${ }^{5-7}$ à morte fetal, ${ }^{8,9}$ pré-eclâmpsia e outros distúrbios hipertensi$\operatorname{vos}^{5,10}$ e abortos espontâneos. ${ }^{5}$ Embora esta associação seja clara, a maioria das gestações com estes desfechos não apresentam valores baixos de PAPP-A, mostrando uma baixa sensibilidade para este marcador. Por outro lado, são poucas as grávidas com valores baixos de PAPP-A que têm estas complicações obstétricas (valor preditivo positivo baixo).

Também se têm estudado complicações obstétricas associadas a valores baixos de $\beta$-HCG como o risco aumentado de recém-nascidos LIG e abortos espontâneos. ${ }^{5}$

\section{CONCLUSÃO}

Concluímos que níveis baixos da PAPP-A e de $\beta$-HCG estiveram relacionados com aumento do risco do nascimento de recém-nascidos leves para a idade gestacional na população estudada. Contudo, são necessários mais estudos que permitam optimizar o rastreio das complicações obstétricas referidas. Os resultados apresentados corroboram a evidência crescente de que os marcadores bioquímicos do primeiro trimestre venham a constituir ferramentas úteis a incluir nesse modelo de rastreio.

\section{CONFLITOS DE INTERESSE}

Os autores declaram não ter quaisquer conflitos de interesse relativamente ao presente artigo.

\section{FONTES DE FINANCIAMENTO}

Não existiram quaisquer fontes externas de financiamento na elaboração do presente trabalho.

Tabela 4 - Estudos publicados sobre a relação da PAPP-A e $\beta$-HCG com recém-nascidos leves para a idade gestacional

\begin{tabular}{|c|c|c|c|c|c|c|}
\hline \multirow[b]{2}{*}{ Estudos } & \multirow[b]{2}{*}{ Total } & \multirow[b]{2}{*}{ Percentil } & \multicolumn{2}{|c|}{ PAPP-A } & \multicolumn{2}{|l|}{$\beta$-HCG } \\
\hline & & & $\begin{array}{c}\text { Valor médio } \\
\text { (MoM) }\end{array}$ & OR & $\begin{array}{c}\text { Valor médio } \\
\text { (MoM) }\end{array}$ & OR \\
\hline Goetzinger et $\mathrm{al}^{1}$ & 2153 & $<\mathrm{P}_{10}$ & & 2,6 & & 1,1 \\
\hline Kirkegaard et $\mathrm{al}^{2}$ & 9450 & $<P_{5}$ & & 2,4 & & 1,8 \\
\hline Ranta et $\mathrm{al}^{4}$ & 2844 & $<\mathrm{P}_{10}$ & 0,79 & 2,6 & 0,90 & 1,7 \\
\hline \multirow[t]{2}{*}{ Spencer et al ${ }^{11}$} & 49801 & $<\mathrm{P}_{10}$ & 0,82 & 2,7 & 0,98 & 1,4 \\
\hline & & $<\mathrm{P}_{3}$ & 0,75 & 3,7 & 0,98 & 1,7 \\
\hline Dugoff et al ${ }^{5}$ & 33395 & $<\mathrm{P}_{10}$ & & 2,5 & & 1,6 \\
\hline \multirow[t]{2}{*}{ Este estudo } & 2305 & $<\mathrm{P}_{10}$ & 1,04 & 2,4 & 0,90 & 1,7 \\
\hline & & $<\mathrm{P}_{3}$ & 0,92 & 3,4 & 0,87 & 3,2 \\
\hline
\end{tabular}




\section{REFERÊNCIAS}

1. Goetzinger KR, Singla A, Gerkowicz S, Dicke JM, Gray DL, Odibo AO. The efficiency of first-trimester serum analytes and maternal characteristics in predicting fetal growth disorders. Prenat Diagn. 2010;30:1138-42.

2. Carbone JF, Tuuli MG, Bradshaw R, Liebsch J, Odibo AO. Efficiency of first-trimester growth restriction and low pregnancy associated plasma protein-A in predicting small for gestational age at delivery. Prenatal Diagn. 2012;32:724-9.

3. Kirkegaard I, Henriksen TB, Uldbjerg N. Early fetal growth, PAPP-A and free $\beta$-hCG in relation to risk of delivering a small-for-gestational age infant. Ultrasound Obstet Gynecol. 2011;37:341-7.

4. Ranta JK, Raatikainen K, Romppanen J, Pulkki K, Heinonen S. Decreased PAPP-A is associated with preeclampsia, premature delivery and small for gestational age infants but not with placental abruption. Eur J Obstet Gynecol Reprod Biol. 2011;157:48-52.

5. Dugoff L, Hobbins JC, Malone FD, Porter TF, Luthy D, Comstock $\mathrm{CH}$, et al. First-trimester maternal serum PAPP-A and free-beta subunit human chorionic gonadotropin concentrations and nuchal translucency are associated with obstetric complications: a population-based screening study (the FASTER Trial). Am J Obstet Gynecol. 2004;191:1446-51.

6. Spencer K, Cowans NJ, Molina F, Kagan KO, Nicolaides KH. First-trimester ultrasound and biochemical markers of aneuploidy and the prediction of preterm or early preterm delivery. Ultrasound Obstet Gynecol. 2008;31(2):147-52.

7. Goetzinger KR, Cahill AG, Macones GA, Odibo AO. Association of first-trimester low PAPP-A levels with preterm birth. Prenat Diagn. 2010;30:309-12.

8. Spencer K, Cowans NJ, Avgidou K, Nicolaides KH. First-trimester ultrasound and biochemical markers of aneuploidy and the prediction of impending fetal death. Ultrasound Obstet Gynecol. 2006;28:637-43.
9. Smith GC, Crossley JA, Aitken DA, Pell JP, Cameron AD, Connor JM, et al. First-trimester placentation and the risk of antepartum stillbirth. JAMA. 2004;292:2249-54.

10. Spencer K, Cowans NJ, Nicolaides KH. Low levels of maternal serum PAPP-A in the first trimester and the risk of pre-eclampsia. Prenat Diagn. 2008;28:7.

11. Spencer K, Cowans NJ, Avgidou K, Molina F, Nicolaides KH. First-trimester biochemical markers of aneuploidy and the prediction of smallfor-gestational age fetuses. Ultrasound Obstet Gynecol. 2008;31:15-9.

12. Smith GC, Stenhouse EJ, Crossley JA, Aitken DA, Cameron AD, Connor JM. Early-pregnancy origins of low birth weight. Nature. 2002;417:916.

13. Rutanen EM. Insulin-like growth factors in obstetrics. Curr Opin Obstet Gynecol. 2000;12:163-8.

14. Krantz D, Goetzl L, Simpson JL, Thom E, Zachary J, Hallahan TW, et al. Association of extreme first-trimester free human chorionic gonadotropin-beta, pregnancy-associated plasma protein A, and nuchal translucency with intrauterine growth restriction and other adverse pregnancy outcomes. Am J Obstet Gynecol. 2004;191:1452-8.

15. Kagan KO, Etchegaray A, Zhou Y, Wright D, Nicolaides KH. Prospective validation of first-trimester combined screening for trisomy 21 . Ultrasound Obstet Gynecol. 2009;34:14-8.

16. Kagan KO, Wright D, Spencer K, Molina FS, Nicolaides KH. First-trimester screening for trisomy 21 by free beta-human chorionic gonadotropin and pregnancy-associated plasma protein-A: impact of maternal and pregnancy characteristics. Ultrasound Obstet Gynecol. 2008;31:493502.

17. Hadlock FP, Harrist RB, Martinez-Poyer J. In utero analysis of fetal growth: a sonographic weight standard. Radiology. 1991;181:129-33. 


\section{Marcadores Bioquímicos do Primeiro Trimestre e Recém-Nascidos Leves para Idade Gestacional Acta Med Port 2014:27:191-195}

Publicado pela Acta Médica Portuguesa, a Revista Científica da Ordem dos Médicos

Av. Almirante Gago Coutinho, 151

1749-084 Lisboa, Portugal.

Tel: +351 218428215

E-mail: submissao@actamedicaportuguesa.com

www.actamedicaportuguesa.com

ISSN:0870-399X | e-ISSN: 1646-0758

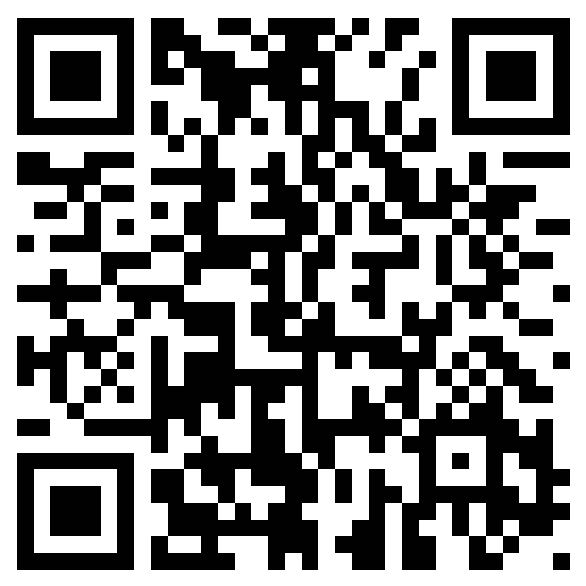

